\title{
COMPUTATIONAL METHODS FOR VOLTERRA-FREDHOLM INTEGRAL EQUATIONS
}

\author{
LECHOSLAW HACCIA \\ Institute of Mathematics, Poznań University of Technology \\ Piotrowo 3A, 60-965 Poznań, Poland, e-mail: lhacia@math.put.poznan.pl
}

\begin{abstract}
Integral equations in space-time play very important role in mechanics and technology. Particular cases of these equations called mixed integral equations or Volterra-Fredholm integral equations arise in the heat conduction theory $[4,6]$ and the diffusion theory. Moreover, a current density in electromagnetism is determined by the Volterra-Fredholm integral equations [4]. Nonlinear counterparts of the equations studied in [1] are mathematical models of the spatio-temporal development of an epidemic (the spread of the disease in the given population). Some initial-boundary problems for a number of partial differential equations in physics are reducible to the considered integral equations [2- 3, 6], In this paper the general theory of these equations is used in the projection methods. Presented methods lead to a system of algebraic equations or to a system of Volterra integral equations. The convergence of studied algorithm is proved, the error estimate is established. The presented theory is illustrated by numerical examples.
\end{abstract}

\section{GENERAL CONSIDERATIONS}

The following integral equation

$$
u(x, t)=f(x, t)+\int_{0}^{t} \int_{M} k(x, t, y, s) u(y, s) d y d s
$$

in space-time is considered, where/is given function in domain $D=M \times[0, T]$ ( $M$ - a compact subset of $n$-dimensional Euclidean space) and $u$ is unknown function in $D$; given kernel $k$ is defined in domain $\Omega=\{(x, t, y, s): x, y \in M, 0 \leq s \leq t \leq T\}$. Integral equations of this type arise in various physical, mechanical and biological problems. The general theory for the considered equations in weighted spaces was presented in [3], Approximate solutions of Volterra-Fredholm integral equations were studied in papers [1-8]

The mixed integral equation (1) can be written in the operator form

$$
u=f+K u \text {, }
$$

where

$$
(K u)(x, t)=\int_{0}^{t} \int_{M} k(x, t, y, s) u(y, s) d y d s
$$

is the integral operator in that a Volterra part plays the dominant role. On virtue this property we can prove the existence and uniqueness of solutions of the equation (1) in the spaces $\mathrm{C}$ and $L^{p} \quad(p \geq 1)[3]$. 


\section{Theorem 1}

Let $f$ and $k$ be continuous functions on $D$ and $\Omega$ respectively. Then the sequence $\left\{u_{n}\right\}$ defined by formula

$$
u_{n}=f+K u_{n-1}, \quad n=1,2,3, \ldots ; \quad u_{0}=f
$$

is uniformly convergent to unique solution of equation (1) given in the form

$$
u(x, t)=f(x, t)+\int_{0}^{t} \int_{M} r(x, t, y, s) f(y, s) d y d s,
$$

where $r$ is the resolvent kernel defined by the formula

$$
r(x, t, y, s)=\sum_{n=1}^{\infty} k_{(n)}(x, t, y, s)
$$

with iterated kernels

$$
\begin{gathered}
k_{(n)}(x, t, y, s)=\iint_{s M} k_{(1)}(x, t, u, v) k_{(n-1)}(u, v, y, s) d u d v \quad \text { for } n=2,3, \ldots \\
k_{(1)}(x, t, y, s)=k(x, t, y, s) .
\end{gathered}
$$

Proof of this theorem is similar as in the case of Volterra integral equation

\section{Remark 1}

A solution of the Volterra-Fredholm integral equation can be presented in the operator form

$$
u=(I-K)^{-1} f=\sum_{n=0}^{\infty} K^{n} f
$$

where

$$
K^{n}=K\left(K^{n-1}\right), \quad n=1,2, \ldots, K^{0}=I, K^{1}=K
$$

\section{GALERKIN TYPE METHOD}

Classic al Galerkin method for integral equation (1) leads to approximate solution of the form

$$
u_{n}(x, t)=\sum_{j=1}^{n} c_{j} \chi_{j}(x, t) \text {, }
$$

where $\left\{\chi_{i}\right\}$ is the orthogonal basis in the space $L^{2}(D)$. Because it is difficult to define such a system we propose the following formula

$$
u_{n}(x, t)=\sum_{i, k=1}^{n} c_{i, k} \varphi_{i}(x) \psi_{k}(t)
$$

where $\left\{\varphi_{i}\right\},\left\{\psi_{k}\right\}$ are orthogonal bases in spaces $L^{2}(D)$ and $L^{2}[0, T]$, respectively. 
Coefficients $c_{k k}(i, k=1,2, \ldots, n)$ are determined by the orthogonality condition in $L^{2}(D)$ of the form

$$
\left(\varepsilon_{n}, \varphi_{i} \psi_{k}\right)=0 \quad(i, k=1,2, \ldots, n)
$$

where

$$
\varepsilon_{n}=u_{n}-f-K u_{n}
$$

is a deviation function.

In practice, we restrict our considerations to the orthonormal basis. Then we get the following system of linear algebraic equations

$$
c_{i k}=f_{i k}+\sum_{j, m=1}^{n} c_{j m} k_{j m, i k} \quad(i, k=1,2, \ldots, n)
$$

where

$$
\begin{gathered}
f_{i k}=\int_{0}^{T} \int_{M} f(x, t) \varphi_{i}(x) \psi_{k}(t) d x d t \\
k_{j m, i k}=\int_{0}^{T} \int_{M}^{T}\left[\int_{0}^{t} \int_{M} k(x, t, y, s) \varphi_{j}(y) \psi_{m}(s) d y d s\right] \varphi_{i}(x) \psi_{k}(t) d x d t .
\end{gathered}
$$

\section{Theorem 2}

Let $\left\{\varphi_{i}\right\},\left\{\psi_{k}\right\}$, are orthonormal complete systems in the spaces $L^{2}(M)$ and $L^{2}[0, T]$, respectively. If $f \in L^{2}(D)$ and $k \in L^{2}(\Omega)$, then the system (6) is uniquely solvable and the sequence defined by the formula (5) converges to the unique solution of the equation (1) in the space $L^{2}(D)$.

The proof is similar as in the case of the Fredholm integral equation and it is based on the Fourier series theory.

\section{Example 1}

$$
u(x, t)=x^{2}\left(e^{-t}-\frac{2}{3} t^{3}\right)+\int_{0}^{t} \int_{-1}^{1} x^{2} t^{2} e^{s} u(y, s) d y d s \quad t \in\langle 0,1\rangle
$$

Relative errors $(n=6)$

\begin{tabular}{rcccccc}
\hline$x \downarrow t$ & 0.0 & 0.2 & 0.4 & 0.6 & 0.8 & 1.0 \\
\hline-1.0 & $.9619 \mathrm{e}-6$ & $.2089 \mathrm{e}-6$ & $.1250 \mathrm{e}-6$ & $.1273 \mathrm{e}-6$ & $.3487 \mathrm{e}-6$ & $.2317 \mathrm{e}-5$ \\
-0.6 & $.9625 \mathrm{e}-6$ & $.2093 \mathrm{e}-6$ & $.1247 \mathrm{e}-6$ & $.1270 \mathrm{e}-6$ & $.3480 \mathrm{e}-6$ & $.2315 \mathrm{e}-5$ \\
-0.2 & $.9685 \mathrm{e}-6$ & $.2146 \mathrm{e}-6$ & $.1208 \mathrm{e}-6$ & $.1266 \mathrm{e}-6$ & $.3432 \mathrm{e}-6$ & $.2300 \mathrm{e}-5$ \\
0.2 & $.9685 \mathrm{e}-6$ & $.2146 \mathrm{e}-6$ & $.1208 \mathrm{e}-6$ & $.1266 \mathrm{e}-6$ & $.3432 \mathrm{e}-6$ & $.2300 \mathrm{e}-5$ \\
0.6 & $.9625 \mathrm{e}-6$ & $.2093 \mathrm{e}-6$ & $.1247 \mathrm{e}-6$ & $.1270 \mathrm{e}-6$ & $.3480 \mathrm{e}-6$ & $.2315 \mathrm{e}-5$ \\
1.0 & $.9619 \mathrm{e}-6$ & $.2089 \mathrm{e}-6$ & $.1250 \mathrm{e}-6$ & $.1273 \mathrm{e}-6$ & $.3487 \mathrm{e}-6$ & $.2317 \mathrm{e}-5$ \\
\hline
\end{tabular}


Dependence of average relative error of a number $(n)$ of basis functions

\begin{tabular}{ccccc}
\hline$n$ & 4 & 5 & 6 & 7 \\
\hline Relative errors & $.337 \mathrm{e}-3$ & $.163 \mathrm{e}-4$ & $.665 \mathrm{e}-6$ & $.328 \mathrm{e}-7$ \\
\hline
\end{tabular}

\section{Example 2}

$$
u(x, t)=e^{x} t^{2}-\frac{2}{3} x^{2} t^{3}+\int_{0}^{t} \int_{-1}^{1} e^{-y} x^{2} u(y, s) d y d s \quad t \in\langle 0,1\rangle
$$

The average relative error dependence on the number $(n)$ of basis functions

\begin{tabular}{ccccc}
\hline$n$ & 4 & 5 & 6 & 7 \\
\hline Relative errors & $.506 \mathrm{e}-2$ & $.549 \mathrm{e}-3$ & $.615 \mathrm{e}-5$ & $.344 \mathrm{e}-8$ \\
\hline
\end{tabular}

\section{GALERKIN-FOURIER TYPE METHOD (GF - METHOD)}

In this section we propose a projection method for the equation (1) leading to solve a system of Volterra linear integral equations. Numerical solution of (1) we search in the form

$$
u_{n}(x, t)=\sum_{j=1}^{n} a_{j}(t) \varphi_{j}(x)
$$

for $(x, t) \in D, D=M \times[0, T]$, where:

$\left\{\varphi_{j}\right\}$ is an orthonormal and complete basis in w $L^{2}(M)$;

$\left\{a_{j}\right\}$ is a solution to a system of the following Volterra integral equations

$$
a_{j}(t)=f_{j}(t)+\sum_{k=1}^{n} \int_{0}^{t} k_{j k}(t, s) a_{k}(s) d s, \quad j=1,2, \ldots, n
$$

with

$$
\begin{gathered}
f_{j}(t)=\int_{M} f(x, t) \varphi_{j}(x) d x, \quad j=1,2, \ldots, n \\
k_{j k}(t, s)=\int_{M} \int_{M} k(x, t, y, s) \varphi_{j}(x) \varphi_{k}(y) d y d x \quad k, j=1,2, \ldots, n .
\end{gathered}
$$

\section{Lemma 1}

If. $f \in L^{2}(D)$ and $k \in L^{2}(\Omega), \Omega=\{(x, t, y, s): 0 \leq s \leq t \leq T ; x, y \in M\}$, then function (9) is a unique solution in the space $L^{2}(D)$ of the equation

$$
u_{n}(x, t)=f_{n}(x, t)+\int_{0}^{t} \int_{M} k_{n}(x, t, y, s) u_{n}(y, s) d y d s
$$


with

$$
\begin{gathered}
f_{n}(x, t)=\sum_{k=1}^{n} f_{k}(t) \varphi_{k}(x) \\
k_{n}(x, t, y, s)=\sum_{j=1}^{n} \sum_{k=1}^{n} k_{j k}(t, s) \varphi_{j}(x) \varphi_{k}(y) .
\end{gathered}
$$

Proof. Putting (14) and (15) in (13) and using linear independence of system $\left\{\varphi_{j}\right\}$ we get

$$
u_{n}(x t)=\sum_{k=1}^{n} u_{k}(t) \varphi_{k}(x)
$$

where

$$
u_{k}(t)=f_{k}(t)+\sum_{j=1}^{n} \int_{0}^{t} \int_{M} k_{j k}(t, s) \varphi_{j}(y) u_{n}(y, s) d y d s, \quad k=1,2, \ldots, n .
$$

By orthonormality of $\left\{\varphi_{k}\right\}$ from (16) and (17) we obtain the Volterra system of integral equations

$$
u_{k}(t)=f_{k}(t)+\sum_{j=1}^{n} \int_{0}^{t} k_{j k}(t, s) u_{j}(s) d s, \quad k=1,2, \ldots, n .
$$

It follows from the assumptions above and the Volterra theory, that this system has a unique solution $\left\{u_{j}\right\}$ in the space $L^{2}[0, T]$ such that $\left.u_{j}(t)=a f t\right)$ for every $j=1,2, \ldots, n$.

The equation (13) can be rewritten in the operator form

$$
u_{n}=f_{n}+K_{n} u_{n},
$$

where $K_{n}$ is the Volterra-Fredholm integral operator of the form (3) determined by the kernel $k_{n}$ defined by the formula (15).

\section{Theorem 3}

If $f \in L^{2}(D)$ and $k \in L^{2}(\Omega)$, then the sequence $\left\{u_{n}\right\}$ defined by the formula (9) converges in the space $L^{2}(D)$ to the unique solution of the equation (1) and the estimated error

$$
\left\|u_{n}-u\right\|_{L^{2}(D)} \leq \frac{c}{1-c \delta_{n}}\left[\left\|f-f_{n}\right\|_{L^{2}(D)}+\|u\|_{L^{2}(D)} \delta_{n}\right]
$$

holds with

$$
c=\left\|(I-K)^{-1}\right\| \quad \text { and } \quad \delta_{n}=\left\|k_{n}-k\right\|_{L^{2}(\Omega)}
$$

$(\|\cdot\|$ is the operator norm). 
Proof. Subtracting (19) and (2) we get

$$
u_{n}-u=f_{n}-f+\left(K_{n}-K\right) u_{n}+K\left(u_{n}-u\right)
$$

Hence

$$
\left\|u_{n}-u\right\|_{L^{2}(D)} \leq\left\|(I-K)^{-1}\right\|\left[\left\|f_{n}-f\right\|_{L^{2}(D)}+\left\|k_{n}-k\right\|_{L^{2}(\Omega)}\left\|u_{n}\right\|_{L^{2}(D)}\right]
$$

Putting $p=2$ in the theorem 2 and remark 1 of the reference [3] we obtain the following estimate

$$
c=\left\|(I-K)^{-1}\right\| \leq \sum_{j=0}^{\infty} \frac{\|k\|_{L^{2}(\Omega)}^{j}}{\sqrt{j !}}
$$

where

$$
\|k\|_{L^{2}(\Omega)}=\left\{\int_{0}^{T} \int_{M}\left[\int_{0}^{1} \int_{M} k^{2}(x, t, y, s) d y d s\right] d x d t\right\}^{\frac{1}{2}} .
$$

Using the theory of Fourier series and properties of the Lebesgue'a integral [7] we have

$$
F_{n}^{2}(t)=\left\|f_{n}(\cdot, t)\right\|_{L^{2}(M)}^{2} \rightarrow 0 \quad \text { for every } \quad t \in[0, T]
$$

and

$$
\left\|F_{n}\right\|_{L^{2}[0, T]}=\left[\int_{0}^{T}\left\|f_{n}(\cdot, t)-f(\cdot, t)\right\|_{L^{2}(M)}^{2} d t\right]^{\frac{1}{2}}=\left\|f_{n}-f\right\|_{L^{2}(D)} \underset{n \rightarrow \infty}{\longrightarrow} 0 .
$$

Similarly

$$
\left\|k_{n}(\cdot, t, \cdot, s)-k(\cdot, t, \cdot, s)\right\|_{L^{2}(M) \times L^{2}(M)}^{2} \rightarrow 0 \quad \text { for every } \quad 0 \leq s \leq t \leq T
$$

and

$$
\delta_{n}=\left\|k_{n}-k\right\|_{L^{2}(\Omega)} \underset{n \rightarrow \infty}{\rightarrow} 0
$$

Then from (21) we get

$$
\left\|u_{n}-u\right\|_{L^{2}(D)} \underset{n \rightarrow \infty}{\longrightarrow} 0
$$

To obtain estimate (20) let us notice

$$
\left\|u_{n}\right\|_{L^{2}(D)} \leq\left\|u_{n}-u\right\|_{L^{2}(D)}+\|u\|_{L^{2}(D)}
$$

where

$$
u=(I-K)^{-1} f
$$

is a unique solution of equation (1). By the above considerations we have 
$\left\|u_{n}-u\right\|_{L^{2}(D)} \leq c\left[\left\|f_{n}-f\right\|_{L^{2}(D)}+\delta_{n}\left\|u_{n}\right\|_{L^{2}(D)}\right] \leq c\left\|f_{n}-f\right\|_{L^{2}(D)}+c \delta_{n}\left\|u_{n}-u\right\|_{L^{2}(D)}+c \delta_{n}\|u\|_{L^{2}(D)}$.

From the above we get the estimate (20).

\section{IMPLEMENTATION OF THE GF METHOD IN MAPLE V}

Let us consider the following integral equation of the Volterra-Fredholm type

$$
u(x, t)=f(x, t)+\int_{0}^{t} \int_{-1}^{1} k(x, t, y, s) u(y, s) d y d s, \quad t \in[0,1]
$$

restart;

with (orthopoly);

$$
[G, H, L, P, T, U]
$$

with (linalg):

Warning, new definition for norm

Warning, new definition for trace

un $:=(k, x) \rightarrow P(k-1, x) *((2 * k-1) / 2)^{\wedge}(1 / 2)$;

$$
\text { un }:=(k, x) \rightarrow P(k-1, x) \sqrt{k-\frac{1}{2}}
$$

wsp : $=\operatorname{proc}(1, \mathrm{~d}, \mathrm{~h})$

local a, ind;

a: $=1$ :

for ind from 0 to 1 do

if ind $<>d$ then

$\mathrm{a}:=\mathrm{a}^{*}(\mathrm{r}-\mathrm{ind}) /(\mathrm{d}-\mathrm{ind})$ :

fi od;

$\mathrm{h} * \operatorname{int}(\mathrm{a}, \mathrm{r}=0.1)$;

end:

$\mathrm{fj}:=(j, t)->\operatorname{int}(f(x, t) * \operatorname{un}(j, x), x=-1 . .1):$

$k j k:=(j, k, t, s)->\operatorname{int}(\operatorname{int}(k(x, t, y, s) * u n(j, x) * u n(k, y), x=-$

1..1), $y=-1 \ldots 1):$

wekG : $=\operatorname{proc}(\mathrm{n}, \mathrm{k}, \mathrm{h})$

local i;

global G;

$\mathrm{G}:=\mathrm{vector}(\mathrm{n})$;

for $i$ from 1 to $n$ do

if $k=0$ then $G[i]:=\operatorname{limit}(f j(i, t), t=0)$

else $G[i]:=f j\left(i, k^{*} h\right): f i$ :

od; op (G) end: 


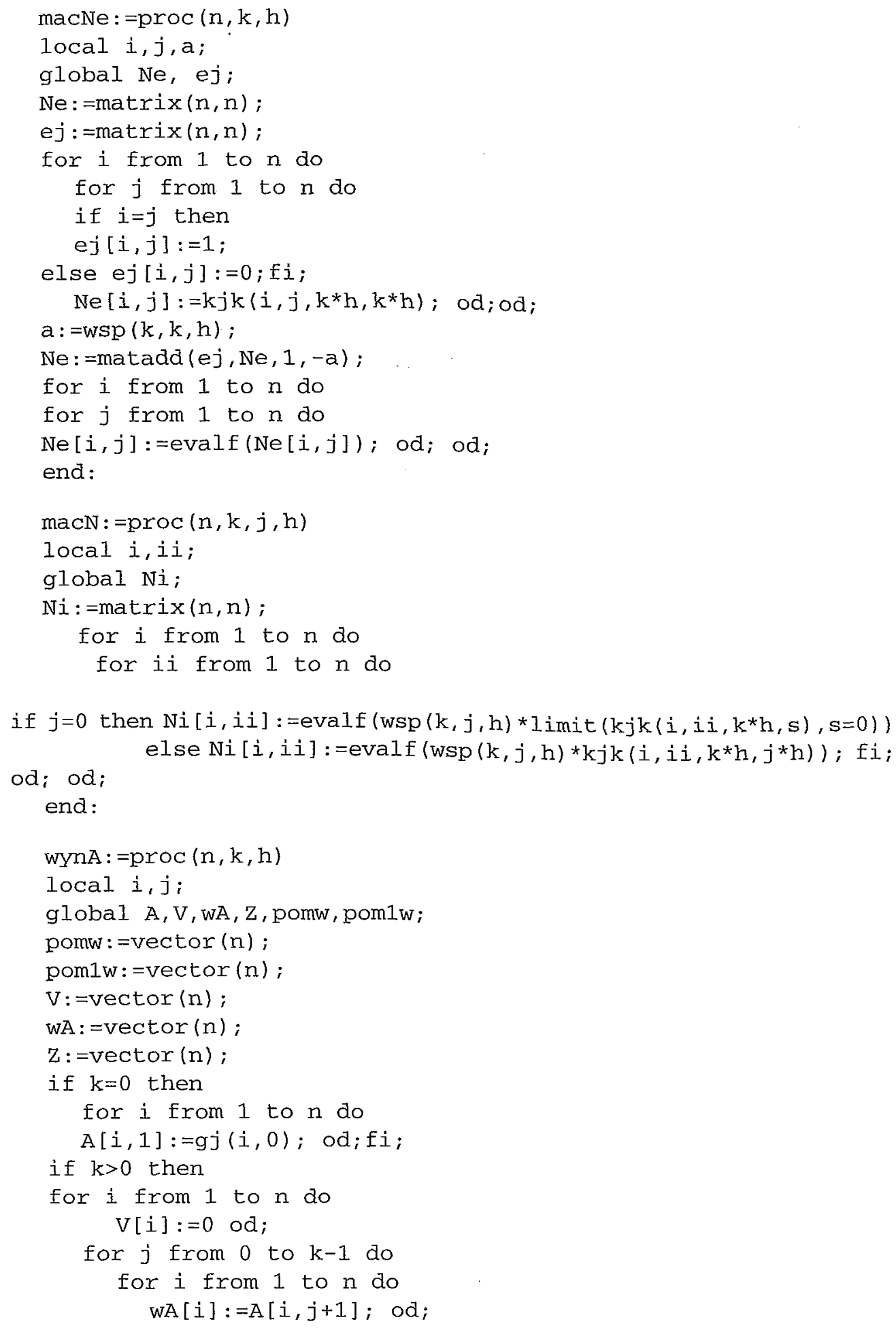




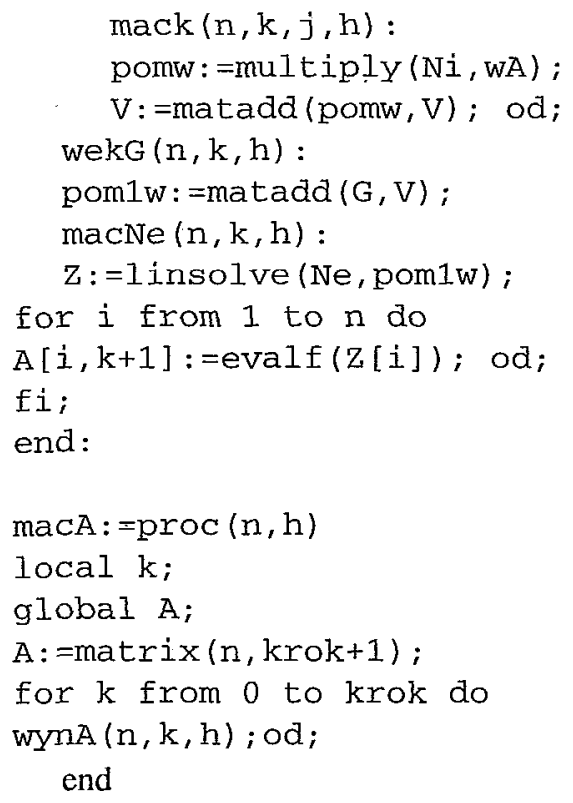

\section{NUMERICAL EXPERIMENTS}

The presented above theory is illustrated by the Legendre polynomials $\left\{\mathrm{P}_{\mathrm{j}}\right\}$ in the orthonormalized form

$$
\varphi_{j}(x)=\frac{P_{j}(x)}{\left\|P_{j}\right\|_{L^{2}(-1,1)}}
$$

forming a complete system in $\mathrm{L}_{(-1,1)}^{2}$.

Functions $\mathrm{a}_{j}(j=1,2, \ldots, n)$ determining the approximate solution (9) of the equation (1) are calculated by the system of Volterra integral equations (10) using the Newton-Cotes quadrature.

\section{Example 3}

Consider the integral equation

$$
u(x, t)=x^{2}\left(e^{-t}-\frac{2}{3} t^{3}\right)+\int_{0}^{t} \int_{-1}^{1} x^{2} t^{2} e^{s} u(y, s) d y d s \quad t \in\langle 0,1\rangle
$$

with an exact solution

$$
u(x, t)=e^{-t} x^{2}
$$

The Galerkin-Fourier method restricted to following basis functions $\left\{\varphi_{\mathrm{j}}\right\}_{j=1,2,3}$ leads to solve the system of $n=3$ integral equations of the Volterra type. 
The relative errors with the step of quadrature formula $h=0.1$ are given below:

\begin{tabular}{llllllll}
\hline$x \mid t$ & \multicolumn{1}{c}{-1} & \multicolumn{1}{c}{-0.6} & \multicolumn{1}{c}{-0.2} & \multicolumn{1}{c}{0.2} & \multicolumn{1}{c}{0.6} & \multicolumn{1}{c}{1} \\
\hline 0.2 & $.1221 \cdot 10^{-9}$ & $.6785 \cdot 10^{-10}$ & $.3053 \cdot 10^{-8}$ & $.3053 \cdot 10^{-8}$ & $.6785 \cdot 10^{-10}$ & $.1221 \cdot 10^{-9}$ \\
0.4 & $.1492 \cdot 10_{-9}$ & $.7459 \cdot 10_{-9}$ & 0 & 0 & $.7459 \cdot 10_{-9}$ & $.1492 \cdot 10^{-9}$ \\
0.6 & $.7288 \cdot 10-9$ & $.7592 \cdot 10-9$ & 0 & 0 & $.7592 \cdot 10-9$ & $.7288 \cdot 10^{-9}$ \\
0.8 & 0 & $.5563 \cdot 10-9$ & $.5564 \cdot 10_{-8}$ & $.5563 \cdot 10_{-8}$ & $.5564 \cdot 10_{-9}$ & 0 \\
1 & $.5436 \cdot 10-9$ & $.1034 \cdot 10-8$ & $.6795 \cdot 10_{-8}$ & $.6795 \cdot 10_{-8}$ & $.1034 \cdot 10_{-8}$ & $.5436 \cdot 10^{-9}$ \\
\hline
\end{tabular}

Experiments for $n=4, n=5$ and $n=6$ give similar errors.

\section{Example 4}

As an approximate solution of the integral equation

$$
u(x, t)=e^{x t}-2 x^{2} t+\int_{0}^{t} \int_{-1}^{1} x^{2} e^{-s y} u(y, s) d y d s
$$

with the exact solution

$$
u(x, t)=e^{x t}
$$

we propose for $n=4$ and $n=6$ basis functions with $h=0.1$.

The tables below give relative errors dependence on $n$ - the number of basis functions $n=4$

\begin{tabular}{rccccc}
\hline$x y$ & \multicolumn{1}{c}{0.2} & \multicolumn{1}{c}{0.4} & 0.6 & \multicolumn{1}{c}{0.8} & \multicolumn{1}{c}{1.0} \\
\hline-1.0 & $.2833 \mathrm{e}-4$ & .0035 & .00214 & .0820 & .02435 \\
-0.6 & $.1020 \mathrm{e}-4$ & .00012 & .00017 & .0255 & .00707 \\
-0.2 & $.1131 \mathrm{e}-5$ & $.5804 \mathrm{e}-4$ & .00029 & .00096 & .00240 \\
0.2 & $.5585 \mathrm{e}-5$ & $.5567 \mathrm{e}-4$ & .00028 & .00088 & .00216 \\
0.6 & $.3000 \mathrm{e}-4$ & $.8005 \mathrm{e}-4$ & .00036 & .00104 & .00231 \\
1.0 & $.6409 \mathrm{e}-5$ & .00017 & .00073 & .00197 & .00411 \\
\hline
\end{tabular}

$n=6$

\begin{tabular}{rlrccc}
\hline$x \backslash t$ & \multicolumn{1}{c}{0.2} & 0.4 & \multicolumn{1}{c}{0.6} & \multicolumn{1}{c}{0.8} & 1.0 \\
\hline-1.0 & .0028 & .00107 & .00011 & $.5638 \mathrm{e}-4$ & .00025 \\
-0.6 & .0010 & .00014 & $.9515 \mathrm{e}-5$ & $.6368 \mathrm{e}-5$ & $.2666 \mathrm{e}-4$ \\
-0.2 & $.5235 \mathrm{e}-4$ & .00024 & $.1871 \mathrm{e}-4$ & $.1348 \mathrm{e}-5$ & $.6988 \mathrm{e}-5$ \\
0.2 & $.5284 \mathrm{e}-4$ & .00020 & $.1647 \mathrm{e}-4$ & $.2333 \mathrm{e}-5$ & $.8495 \mathrm{e}-5$ \\
0.6 & $.7976 \mathrm{e}-4$ & .8283 & $.7821 \mathrm{e}-5$ & $.3284 \mathrm{e}-5$ & $.1078 \mathrm{e}-4$ \\
1.0 & .00018 & .00047 & $.3073 \mathrm{e}-4$ & $.1190 \mathrm{e}-4$ & $.3961 \mathrm{e}-4$ \\
\hline
\end{tabular}


In the following examples the error dependence on the number of the basis functions, $n$, and on a step of the quadrature formula, $h$, are presented.

\section{Example 5}

$$
u(x, t)=e^{x} t^{2}-\frac{2}{3} x^{2} t^{3}+\int_{0}^{t} \int_{-1}^{1} x^{2} e^{-y} u(y, s) d y d s
$$

$n=7, h=0.1$

\begin{tabular}{rlllll}
\hline$x t$ & \multicolumn{1}{c}{2} & \multicolumn{1}{c}{0.4} & \multicolumn{1}{c}{0.6} & \multicolumn{1}{c}{0.8} & 1 \\
\hline-1.0 & .002951504540 & .0009316754594 & .0005217387064 & .0003011446842 & .0003494498724 \\
-0.8 & .001471686767 & .0004491672421 & .0002470762282 & .0001888029023 & .0001057247083 \\
-0.6 & .0007038132108 & .0002215284454 & .0001246720567 & .00007907573039 & .00007494071325 \\
-0.4 & .0002628464290 & .00008236104231 & .00004531180795 & .00002096846684 & .00003459125934 \\
-0.2 & .00004535992933 & .00001150265867 & $.4844021350 \cdot 10^{-5}$ & $.5303752423 \cdot 10^{-5}$ & $.1050509214 \cdot 10^{-5}$ \\
0.0 & $.894174894 \cdot 10^{-5}$ & $.395049370 \cdot 10^{-5}$ & $.231927762 \cdot 10^{-5}$ & $.595781079 \cdot 10^{-5}$ & $.897942294 \cdot 10^{-5}$ \\
0.2 & .00003480231055 & .00001196486759 & $.7585095423 \cdot 10^{-5}$ & $.7079740259 \cdot 10^{-5}$ & $.3106367965 \cdot 10^{-5}$ \\
0.4 & .0001187963372 & .00003759948178 & .00002101366789 & $.9492307633 \cdot 10^{-5}$ & .00001581455058 \\
0.6 & .0002090352867 & .00006382763731 & .00003462899861 & .00002117429978 & .00001982262718 \\
0.8 & .0002982311963 & .00009189269894 & .00005103222412 & .00003985061584 & .00002286854186 \\
1.0 & .0003949166083 & .0001213382348 & .00006597568595 & .00003491359138 & .00004189697059 \\
\hline
\end{tabular}

$n=7, h=0.05$

\begin{tabular}{rlllll}
\hline$x \&$ & \multicolumn{1}{c}{2} & \multicolumn{1}{c}{0.4} & \multicolumn{1}{c}{0.6} & 0.8 & 1 \\
\hline-1.0 & .0002885010120 & .0001139410232 & .00006799026720 & .00002046033564 & .0001577615308 \\
-0.8 & .00007630515338 & .00002068412536 & $.9316806904 \cdot 10^{-5}$ & .00002028007483 & $.5287604116 \cdot 10^{-5}$ \\
-0.6 & .00006118947740 & .00002419606550 & .00001517449982 & $.1458964314 \cdot 10^{-5}$ & .00002869052770 \\
-0.4 & .00002900805438 & .00001055532387 & $.5467117942 \cdot 10^{-5}$ & $.7280818877 \cdot 10^{-5}$ & .00001776675876 \\
-0.2 & $.2502936280 \cdot 10^{-5}$ & $.3195244098 \cdot 10^{-5}$ & $.3312336631 \cdot 10^{-5}$ & $.4851241178 \cdot 10^{-5}$ & $.4488635838 \cdot 10^{-5}$ \\
0.0 & $.894204894 \cdot 10^{-5}$ & $.395179370 \cdot 10^{-5}$ & $.232097762 \cdot 10^{-5}$ & $.595061079 \cdot 10^{-5}$ & $.897332294 \cdot 10^{-5}$ \\
0.2 & $.2718872275 \cdot 10^{-5}$ & $.2112568726 \cdot 10^{-5}$ & $.2117725166 \cdot 10^{-5}$ & $.3199340269 \cdot 10^{-5}$ & $.8017227680 \cdot 10^{-5}$ \\
0.4 & .00001372598260 & $.5335092700 \cdot 10^{-5}$ & $.3110294603 \cdot 10^{-5}$ & $.3200914650 \cdot 10^{-5}$ & $.8254815171 \cdot 10^{-5}$ \\
0.6 & .00001548074193 & $.4392266667 \cdot 10^{-5}$ & $.1648971310 \cdot 10^{-5}$ & $.2203420906 \cdot 10^{-5}$ & $.5892338990 \cdot 10^{-5}$ \\
0.8 & .00001650850702 & $.5383449634 \cdot 10^{-5}$ & $.3029424842 \cdot 10^{-5}$ & $.5826443772 \cdot 10^{-5}$ & $.2590640238 \cdot 10^{-5}$ \\
1.0 & .00003451827128 & .00001066991321 & $.4567512395 \cdot 10^{-5}$ & $.8610915097 \cdot 10^{-5}$ & .00001595477458 \\
\hline
\end{tabular}


$n=6, h=0.1$

\begin{tabular}{clllll}
\hline$x y$ & \multicolumn{1}{c}{2} & \multicolumn{1}{c}{0.4} & \multicolumn{1}{c}{0.6} & \multicolumn{1}{c}{0.8} & \multicolumn{1}{c}{1} \\
\hline-1.0 & .002604990238 & .0006284749356 & .0002329752778 & .00008457112181 & $.2932852123 \cdot 10^{-5}$ \\
-0.8 & .001582839876 & .0005464263584 & .0003397031542 & .0002582730042 & .0002168757585 \\
-0.6 & .0006638395158 & .0001865514741 & .00009136011387 & .00005409212250 & .00003496520962 \\
-0.4 & .0002071956364 & .00003366659415 & $.1064235831 \cdot 10^{-5}$ & .00001381306874 & .00002106109221 \\
-0.2 & .00005790561650 & .00002248013113 & .00001529844682 & .00001314513134 & .00001149398708 \\
0.0 & .000030894341 & .000030906185 & .000030876664 & .000030855667 & .000030855667 \\
0.2 & .00004321193615 & .00001932328743 & .00001459290639 & .00001233597374 & .00001151519530 \\
0.4 & .00009379082420 & .00001571965583 & $.1755681782 \cdot 10^{-5}$ & $.6136043716 \cdot 10^{-5}$ & $.9191662880 \cdot 10^{-5}$ \\
0.6 & .0001969954452 & .00005329277601 & .00002459564492 & .00001364938170 & $.7782236866 \cdot 10^{-5}$ \\
0.8 & .0003206726218 & .0001115289759 & .00006973327798 & .00005387638752 & .00004530955186 \\
1.0 & .0003480209970 & .00008030450600 & .00002689580554 & $5603546948 \cdot 10^{-5}$ & $.4999008513 \cdot 10^{-5}$ \\
\hline
\end{tabular}

$n=6, h=0.05$

\begin{tabular}{clllll}
\hline$x y$ & \multicolumn{1}{c}{2} & \multicolumn{1}{c}{0.4} & \multicolumn{1}{c}{0.6} & \multicolumn{1}{c}{0,8} & \multicolumn{1}{c}{1} \\
\hline-1.0 & .00005801396968 & .0001892595008 & .0002207724063 & .0002370292261 & .0001887848469 \\
-0.8 & .0001874582622 & .0001179429634 & .0001019442894 & .00008975191536 & .0001164250785 \\
-0.6 & .00002121579588 & .00001078090582 & .00001813691663 & .00002352450122 & .00001128925790 \\
-0.4 & .00002664285010 & .00003813921755 & .00004090863578 & .00004206305375 & .00003788518999 \\
-0.2 & .00001004265929 & $.7782304700 \cdot 10^{-5}$ & $.7142428123 \cdot 10^{-5}$ & $.7355300578 \cdot 10^{-5}$ & $.8058058983 \cdot 10^{-5}$ \\
0.0 & .000030894141 & .000030904885 & .000030875864 & .000030847667 & .000030864167 \\
0.2 & .00001112843647 & $.9471039744 \cdot 10^{-5}$ & $.9125763559 \cdot 10^{-5}$ & $.8454934113 \cdot 10^{-5}$ & $.9212023820 \cdot 10^{-5}$ \\
0.4 & .00001127958065 & .00001654477515 & .00001772767477 & .00001882958022 & .00001675121731 \\
0.6 & $.3440900423 \cdot 10^{-5}$ & $.6142594648 \cdot 10^{-5}$ & $.8384229942 \cdot 10^{-5}$ & $.9728296119 \cdot 10^{-5}$ & $.6149341031 \cdot 10^{-5}$ \\
0.8 & .00003894993265 & .00002501967038 & .00002173059103 & .00001985256647 & .00002502890933 \\
1.0 & .00001237743192 & .00003036381558 & .00003451226581 & .00003792032722 & .00003094517762 \\
\hline
\end{tabular}

Example 6

$n=5, h=0.1$

\begin{tabular}{clllll}
\hline$x v$ & \multicolumn{1}{c}{2} & \multicolumn{1}{c}{0.4} & \multicolumn{1}{c}{0.6} & \multicolumn{1}{c}{0.8} & \multicolumn{1}{c}{1} \\
\hline-1.0 & .002343885871 & .0024207066374 & .002416741450 & .002402087346 & .002403449668 \\
-0.8 & .001219091966 & .001153895062 & .001158566256 & .001169497521 & .001168305460 \\
-0.6 & .0005981874547 & .0005445509830 & .0005485517766 & .0005573862628 & .0005564015464 \\
-0.4 & .0001834771533 & .0002270142114 & .0002239587437 & .0002165489282 & .0002173411938 \\
-0.2 & .0002425828288 & .0002778713676 & .0002755868489 & .0002693541824 & .0002699903908 \\
0.0 & .0001143370838 & .00008556305180 & .00008735533923 & .00009251034134 & .000091995333 \\
0.2 & .0003874699704 & .0003638093062 & .0003653302282 & .0003695006930 & .0003690765074 \\
0.4 & .0003164039567 & .0002968338064 & .0002981931269 & .0003015138084 & .0003011605999 \\
0.6 & $.1333389750 \cdot 10^{-5}$ & .00001749322625 & .00001629760012 & .00001364321665 & .00001393727840 \\
0.8 & .0001502976117 & .0001634605285 & .0001625145636 & .0001603074217 & .0001605471728 \\
1.0 & .0004973996422 & .0004870116182 & .0004875667785 & .0004895604622 & .0004893743854 \\
\hline
\end{tabular}


$n=5, h=0.05$

\begin{tabular}{clllll}
\hline$x V$ & \multicolumn{1}{c}{2} & \multicolumn{1}{c}{0.4} & \multicolumn{1}{c}{0.6} & \multicolumn{1}{c}{0.8} & 1 \\
\hline-1.0 & .002724038230 & .002724449388 & .002726571021 & .002725269276 & .002635240278 \\
-0.8 & .0009078495393 & .0009052113166 & .0009048992582 & .0009048985352 & .0009785313593 \\
-0.6 & .0003433637081 & .0003409459530 & .0003408668041 & .0003407509362 & .0004010276542 \\
-0.4 & .0003921091913 & .0003937119110 & .0003939968176 & .0003939149322 & .0003445505776 \\
-0.2 & .0004133962943 & .0004143519008 & .0004148022491 & .0004145691844 & .0003741406254 \\
0.0 & .00002551315345 & .00002617775784 & .00002662459027 & .00002638164668 & $.6724333 \cdot 10^{-5}$ \\
0.2 & .0002729702802 & .0002723236690 & .0002720113546 & .0002721601662 & .0002992625173 \\
0.4 & .0002226595392 & .0002219317016 & .0002217900954 & .0002218181254 & .0002440017393 \\
0.6 & .00007808482727 & .00007881788282 & .00007885111172 & .00007889252310 & .00006073499543 \\
0.8 & .0002131363740 & .0002136689106 & .0002137290472 & .0002137290356 & .0001988619029 \\
1.0 & .0004459516151 & .0004459044718 & .0004456362241 & .0004458225440 & .0004580049376 \\
\hline
\end{tabular}

$n=6, h=0.1$

\begin{tabular}{cccccl}
\hline$x \| t$ & 2 & 0.4 & 0.6 & 0.8 & 1 \\
\hline-1.0 & .0006681236336 & .0005728582112 & .0005857252545 & .0005877641316 & .0005931072997 \\
-0.8 & .0002339453603 & .0001748791371 & .0001767390503 & .0001918761365 & .0001885199628 \\
-0.6 & .0002900594076 & .0002383758272 & .0002415336087 & .0002517123882 & .0002500613044 \\
-0.4 & .0002639255834 & .0002176829060 & .0002220957504 & .0002276595554 & .0002278734767 \\
-0.2 & .0001736311681 & .0001358203922 & .0001393623016 & .0001438733842 & .0001441716672 \\
0.0 & .0001143626488 & .00008561987526 & .00008744445973 & .00009262495074 & .000092119333 \\
0.2 & .0001085152463 & .00008659647292 & .00008732742593 & .00009269364010 & .00009165842272 \\
0.4 & .0001154072219 & .00009709469108 & .00009788740171 & .0001020717206 & .0001012789926 \\
0.6 & .00009150105526 & .00007478732926 & .00007627231576 & .00007854978302 & .00007846673466 \\
0.8 & .00004862303190 & .00003425044264 & .00003579301950 & .00003717392628 & .00003737954102 \\
1.0 & .00008978729217 & .00008191851548 & .00008129262232 & .00008501239032 & .00008392573340 \\
\hline
\end{tabular}

$n=6, h=0.05$

\begin{tabular}{clllll}
\hline$x V$ & \multicolumn{1}{c}{2} & \multicolumn{1}{c}{0.4} & \multicolumn{1}{c}{0.6} & \multicolumn{1}{c}{0.8} & \multicolumn{1}{c}{1} \\
\hline-1.0 & .0002879712750 & .0002691142740 & .0002758933314 & .0002645993500 & .0003611481565 \\
-0.8 & .00007729706655 & .00007380536532 & .00007692987343 & .00007208808487 & $.1392121252 \cdot 10^{-5}$ \\
-0.6 & .00003523566100 & .00003477017734 & .00003384705971 & .00003508855698 & .00009457444082 \\
-0.4 & .00005529354542 & .00005098469886 & .0000520568579 & .00005030296280 & .0001005715998 \\
-0.2 & $.2817702621 \cdot 10^{-5}$ & $.6605565040 \cdot 10^{-6}$ & $.1458445038 \cdot 10^{-6}$ & $.1333912417 \cdot 10^{-5}$ & .00003994570569 \\
0.0 & .00002548758852 & .00002612127462 & .00002653633502 & .00002626072852 & $.6786333 \cdot 10^{-5}$ \\
0.2 & $.5984443762 \cdot 10^{-5}$ & $.4889442888 \cdot 10^{-5}$ & $.5992156005 \cdot 10^{-5}$ & $.4641721588 \cdot 10^{-5}$ & .00002179367136 \\
0.4 & .00002166280446 & .00002219235834 & .00002148379014 & .00002238026658 & .00004407857216 \\
0.6 & .00001474961774 & .00001346248596 & .00001371832929 & .00001330393889 & .00003163499131 \\
0.8 & .00001421573036 & .00001595809250 & .00001542185295 & .00001624485284 & $.9630474879 \cdot 10^{-6}$ \\
1.0 & .00003833926503 & .00004081124370 & .00003936161313 & .00004127679308 & .00005253347704 \\
\hline
\end{tabular}




\section{CONCLUSIONS}

Comparing the examples 1 and 3 we notice that for $n=3$ the method of the Galerkin-Fourier type gives better results than the Galerkin type method for $n=4,5,6,7$. The G-F method is dependent on the precision of solving the system of the Volterra integral equations; in the example 2 better results are obtained than in the example 5. Increasing the number of the basis functions, $n$, and the step of the quadrature formula, $h$, gives results of increasing accuracy.

\section{References}

[1] H. Brunner, SIAM J. Numer. Anal., 27, 987-1000 (1990).

[2] L. Hacia, Zeit. Ang. Math. Mech., 76, 415-416 (1996).

[3] L. Hącia, Demon. Math. 32, 795-805 (1999).

[4] L. Hącia, R. Nawrowski, Computer Applications in Electrical Engineering - Monograph ZKwE, 60-78 (2002).

[5] J. P. Kauthen, Numer. Math., 56, 409-429 (1989).

[6] A. Piskorek, Integral equations - theory and applications, WNT, Warszawa 1997 (in Polish).

[7] W. I. Tivončuk, Diff. Uravn., 2(9), 1228-1238 (1966) - in Russian.

[8] I. N. Tukalevska, II Sc. Conf. of Young Ukr. Math., Naukova Dumka, Kiev 1966, 609-613 - in Ukrainian.

[9] I. H. Sloan, B. J. Burn, J. Int. Equ., 1, 77-94 (1979).

[10] I. H. Sloan., J. Int. Equ., 265-274 (1980). 